Enero 2010 | ICTSD Programa sobre competitividad y desarrollo sostenible
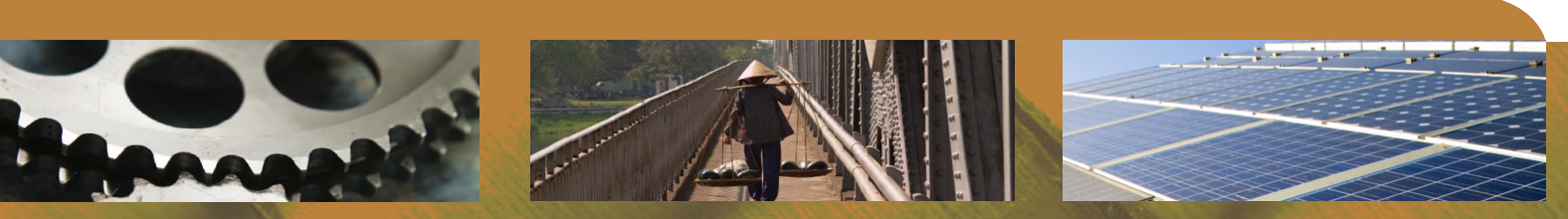

\title{
Hoja de ruta para el sector textil y de confecciones y el desarrollo sostenible en Nicaragua
}

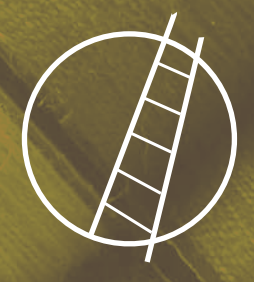

Por Ana Victoria Portocarrero Lacayo

Consultora, NITAPLAN

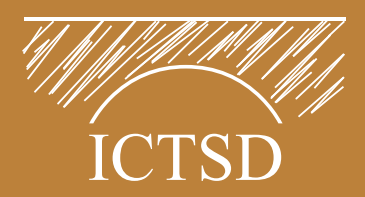

International Centre for Trade and Sustainable Development

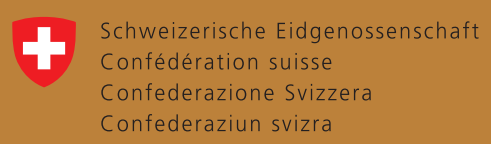

SChweizerische Eidgenossenschaft

Confédération suisse

Confederazione Svizzera

Confederaziun svizra

Swiss Confederation 


\section{Hoja de ruta para el sector textil y de confecciones y el desarrollo sostenible en Nicaragua}

Por Ana Victoria Portocarrero Lacayo

Consultora, NITAPLAN

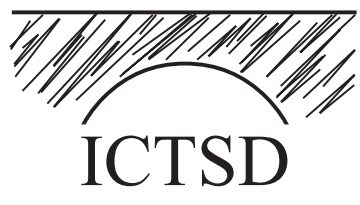

International Centre for Trade and Sustainable Development
Schweizerische Eidgenossenschaft

Confédération suisse

Confederazione Svizzera

Confederaziun svizra

Swiss Confederation

Federal Department of Economic Affairs FDEA State Secretariat for Economic Affairs SECO

Documento de Trabajo No. 2 
Publicado por

El Centro Internacional para el Comercio y el Desarrollo Sostenible - International Centre for Trade and Sustainable Development (ICTSD)

International Environment House 2

7 Chemin de Balexert, 1219 Ginebra, Suiza

Tel: +41229178492

Fax: +41229178093

E-mail: ictsd@ictsd.ch

Internet: www.ictsd.org

Presidente Ejecutivo:

Ricardo Meléndez-Ortiz

Gerente de Programa:

Gloria Carrión

Gerente de Programa Junior: Paolo Ghisu

\section{Reconocimientos:}

La autora agradece el apoyo técnico del ICTSD para la elaboración del presente documento. Este documento ha sido producido con el apoyo financiero de la Secretaría del Estado Suizo para los Asuntos Económicos (SECO).

Para mayor información sobre el programa de ICTSD sobre Competitividad y Desarrollo Sostenible: http://www.ictsd.org.

El ICTSD agradece todos los comentarios sobre este documento. Por favor enviarlos a: Paolo Ghisu, pghisu@ictsd.ch.

Citación: Portocarrero, A. (2010), El sector textil y confección y el desarrollo sostenible en Nicaragua, Documento de Trabajo No.2, International Centre for Trade and Sustainable Development, Geneva, Switzerland.

Copyright @ ICTSD, 2010. El ICTSD autoriza la utilización y reproducción de este documento para actividades académicas y/o sin fines de lucro.

Las opiniones expresadas en este documento corresponden a los autores y no reflejan las opiniones de ICTSD y de otras instituciones donantes.

Este trabajo está bajo la Licencia Creative Commons Reconocimiento-No comercial-Sin obras derivadas 3.0. Para ver una copia de esta licencia, visitar: http://creativecommons.org/licenses/ by-nc-nd/3.0/es/ o envíe una carta a Creative Commons, 171 Second Street, Suite 300, San Francisco, California, 94105, USA.

ISSN 1995-6932 


\section{TABLA DE CONTENIDOS}

LISTA DE ACRÓNIMOS iv

1. INTRODUCCIÓN 1

2. AGENDA DE NEGOCIACIONES COMERCIALES 2

2.1 Enfoque de la política comercial de Nicaragua 2

2.2 Diversificación de mercados y productos 2

2.3 Instrumentos comerciales 3

3. AYUDA PARA EL COMERCIO 8

3.1 Infraestructura de energía y transporte $\quad 8$

$\begin{array}{ll}3.2 \text { Estructura regulatoria } & 8\end{array}$

3.3 ¿¿Formación o capacitación?? $\quad 9$

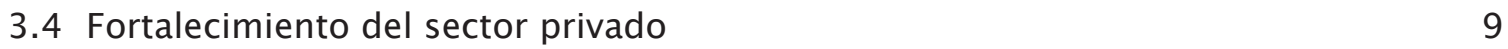

4. POLÍTICAS ESTRUCTURALES 11

4.1 Articulación en política económica 11

4.2 Política de formación y desarrollo 12

$\begin{array}{ll}4.3 \text { Infraestructura } & 12\end{array}$

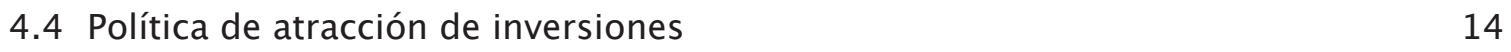

4.5 Participación inclusiva en el comercio 16

$\begin{array}{ll}4.6 \text { Políticas de financiamiento } & 18\end{array}$

5. CONCLUSIONES GENERALES 20

NOTAS 21

REFERENCIAS BIBLIOGRÁFICAS $\quad 22$ 


\section{LISTA DE ACRÓNIMOS}

ADPIC Acuerdo sobre Aspectos de Propiedad Intelectual Relacionados con el Comercio

ANITEC Asociación Nicaragüense de la Industria Textil y la Confección

ASMC Subsidios y Medidas Compensatorias

BIT Tratados Bilaterales de Inversión

CAFTA Central American Free Trade Agreement

CANISLAC Cámara Nicaragüense del Sector Lácteo

CEPAL Comisión Económica para América Latina

DGA Dirección General de Aduanas

EDSAC Estrategias de Desarrollo Sostenible Apoyadas en el Comercio

ICTSD International Center for Trade and Sustainable Development

IDB Inter-American Development Bank

IED Inversión Extranjera Directa

INPYME Instituto Nicaragüense de Apoyo a la Pequeña y Mediana Empresa

MARENA Ministerio de Ambiente y los Recursos Naturales

MIC Acuerdo de Medidas de Inversión Relativas al Comercio

MIFIC Ministerio de Fomento, Industria y Comercio

MIPYMES Micro, Pequeñas y Medianas Empresas

MITRAB Ministerio del Trabajo

OECD Organisation for Economic Cooperation and Development

OMC Organización Mundial del Comercio

PIB Producto Interno Bruto

PND Plan Nacional de Desarrollo

PNDH Plan Nacional de Desarrollo Humano

RZF Régimen de Zonas Francas

SME Metros Cuadrados Equivalentes

TPL Trade Preference Level

UE Unión Europea

WTO World Trade Organization 


\section{INTRODUCCIÓN}

Según el enfoque de las Estrategias de Desarrollo Sostenible Apoyadas en el Comercio (Edsac), son dos los pilares para garantizar un desarrollo sostenible con equidad a partir del comercio: el diseño de estrategias del lado de la oferta -que promuevan complementariedad, innovación, avance tecnológico, encadenamientos entre sectores económicos, aumento del capital humano y social y aseguramiento de la sostenibilidad ambiental-y la creación o fortalecimiento de los marcos institucionales habilitantes para que estas estrategias puedan implementarse.

La viabilidad y eficacia de las estrategias propuestas en este documento dependen directamente de las condiciones institucionales del país. Entre los marcos institucionales pertinentes se contemplan aspectos macroeconómicos tales como políticas de generación de empleo, de crecimiento económico y de estabilidad de precios; así como la política industrial, comercial, de inversiones y en general el clima de negocios del país.

En el documento diagnóstico "Proyecto textil y confección y desarrollo sostenible en Nicaragua" finalizado en mayo de 2009, se analiza la situación actual del sector textil y de confecciones de Nicaragua, según el enfoque de las Edsac. De los hallazgos de ese diagnóstico se ha desprendido una serie de estrategias de reconversión del sector, en función de una estrategia de desarrollo sostenible, que se exponen en el documento "Estrategias para la reconversión del la industria textily confecciones en Guatemala y Nicaragua" (Burga, 2009) -en adelante "documento de estrategias". A continuación se plantea una Hoja de Ruta, o recomendaciones acerca de las condiciones que se deben garantizar en el país para llevar a cabo las estrategias propuestas, así como de los posibles esquemas de financiamiento para el logro de estas condiciones.

La Hoja de Ruta expone lo que a juicio del equipo de investigación $-y$ a través de discusiones con diversos actores del sectordeberán ser los espacios que en materia comercial deben asegurarse en las actuales y futuras negociaciones comerciales en las que Nicaragua participe; algunas recomendaciones de carácter general y otras específicas para el sector textil; la vinculación que existe entre las estrategias y los instrumentos planteados en el documento de estrategias (Burga, 2009); el esquema de financiamiento disponible a través de la Organización Mundial del Comercio (OMC) para los países con obstáculos del lado de la oferta, titulado Ayuda para el Comercio; y finalmente las políticas estructurales que deben asegurarse para el desarrollo sostenible y equitativo del país a través del comercio. 


\section{AGENDA DE NEGOCIACIONES COMERCIALES}

El enfoque de las Edsac propone una serie de instrumentos de política comercial que han sido identificados como elementos clave para asegurar espacios a nivel nacional a fin de diseñar e implementar políticas de fomento de la oferta exportable del país, así como instrumentos que propicien un esquema de crecimiento de las exportaciones y de apertura comercial en pro de un modelo de desarrollo sostenible.

Entre estos instrumentos se encuentran el empleo de incentivos y requisitos de desempeño, la promoción focalizada de la inversión extranjera, limitaciones a las importaciones, preferencias en las compras públicas, y protección a la propiedad intelectual (Corrales, 2007).

A lo largo de la investigación sobre el sector, realizada en Nicaragua entre 2008 y 2009, se generaron diálogos con diversos actores para retroalimentar y afinar los principales hallazgos del diagnóstico. Uno de los temas más discutidos fue el de los acuerdos comerciales firmados por Nicaragua, y en concreto la manera en que están funcionando en el país los diversos instrumentos de política comercial.

A continuación se presentan los principales elementos que en términos de negociaciones comerciales deben asegurarse a fin de garantizar los espacios para implementar debidamente las estrategias de desarrollo sostenible identificadas.

\subsection{Enfoque de la Política Comercial de Nicaragua}

Al analizar el enfoque de la política comercial de Nicaragua desde la década de los 90 , se aprecia que en términos generales el objetivo central de la política comercial del país ha sido una creciente inserción a la economía internacional bajo un esquema de atracción de inversión extranjera directa (IED), apertura comercial y liberalización de mercados, que ha respondido a una estrategia de crecimiento del país.
Al contrastar la política comercial de Nicaragua con el enfoque de las Edsac -que propone justamente superar esa perspectiva del comercio centrada únicamente en el acceso a mercados y en atraer IED, y pasar a enfatizar aspectos tales como la transformación productiva y el acceso al conocimiento, a través de la transferencia de tecnología, por ejemplo- se evidencia la necesidad de transformar el enfoque de política comercial del país hacia uno más integral, que contemple las necesidades del país en términos de desarrollo, y no únicamente de crecimiento.

Por consiguiente, además de los cambios específicos en materia de negociaciones comerciales que se abordarán en la presente sección, se sugiere una reformulación de la política comercial del país, en función de objetivos de desarrollo sostenible con equidad. El primer gran cambio debe estar visible en la estrategia general de comercio exterior del país, para luego formular sus instrumentos específicos.

\subsection{Diversificación de Mercados y Pro- ductos}

La oferta exportable del sector textil y de confecciones se encuentra muy poco diversificada: la mayor parte de la producción consiste en pantalones y camisetas de algodón para hombres y mujeres. ${ }^{1}$ Por otro lado, los mercados de exportación se encuentran también poco diversificados, siendo el de Estados Unidos el que absorbe la mayor parte de las exportaciones del país.

En términos de política comercial exterior es de suma importancia procurar la diversificación de mercados a fin de que el sector exportador del país sea menos dependiente del mercado de Estados Unidos. También hay que potenciar la diversificación de la oferta exportable del sector, de modo que apunte hacia nichos de mercado más variados, hacia productos con una clara diferenciación que no compitan directamente con los 
productos asiáticos, que potencie las ventajas estratégicas que ofrece Nicaragua -como lo es su cercanía con Estados Unidos, México y Canadá- y que se oriente hacia prendas de vestir de mayor valor agregado y hacia una producción ambientalmente sostenible.

\subsection{Instrumentos Comerciales}

Proponemos aquí una agenda de negociación de tratados de libre comercio bilaterales para Nicaragua. Se desarrolla a partir de cuatro insumos principales: un análisis realizado por Werner Corrales (2007) sobre los espacios para políticas que han desaparecido y los que aún existen en los principales acuerdos en que participan los países de América Latina y el Caribe; el documento diagnóstico sobre el sector textil y de confecciones y el desarrollo sostenible en Nicaragua (Portocarrero, A., 2009); las estrategias de reconversión del sector, tanto a nivel de temas estratégicos como de marcos institucionales habilitantes, presentadas por Burga (2009); y finalmente la discusión con el sector privado, el gobierno, los especialistas y las organizaciones de la sociedad civil vinculadas con el sector sobre los documentos antes citados y el contraste de los mismos con la experiencia de Nicaragua desde la firma del Cafta.

\subsubsection{Empleo de incentivos y requisitos de desempeño}

Se deben mantener los espacios para la utilización de mecanismos de incentivos, desincentivos y requisitos de desempeño - condicionados o no- que apunten hacia la implementación de las estrategias de desarrollo sostenible del sector (encadenamientos, complementariedades, derrames tecnológicos y otras).

Habida cuenta de que el Acuerdo sobre Subsidios y Medidas Compensatorias de la OMC (ASMC) prohíbe utilizar incentivos de manera selectiva y restringe aún más el Acuerdo de Medidas de Inversión Relativas al Comercio
(MIC) utilizado en los Tratados Bilaterales de Inversión (BIT), la propuesta es que se mantenga el espacio para utilizar incentivos aplicados horizontalmente, cuyo criterio de aplicación no es sectorial. Así, el sector textil y de confecciones no sería el único beneficiado por este instrumento.

A la vez se debe oficializar el mecanismo de subsidios "no recurribles", en los que se incluyen las áreas de investigación y desarrollo, el desarrollo regional, la introducción de tecnologías ambientales y el financiamiento de fondos de capital de riesgo.

\subsubsection{Promoción focalizada de la inversión} extranjera

Con el fin de que se cumpla la estrategia de atracción de inversiones que promueva encadenamientos y complementariedades en los sectores económicos, y que genere mayor valor agregado y más transferencia tecnológica y de conocimientos, debe garantizarse que no se restrinja este espacio en los compromisos comerciales que el país adquiere.

Corrales (2007) señala que actualmente no existen restricciones en materia de focalización de la promoción de inversiones hacia determinados sectores productivos o hacia actividades específicas. Señala asimismo que se permite el uso de algunos subsidios para incentivar inversiones en algunas actividades en las que los gobiernos tengan especial interés y que coincidan con estrategias de desarrollo nacionales. Por tal motivo se debe mantener el espacio que aún tienen los gobiernos para atraer inversión extranjera focalizada, de modo que permita vincular la inversión con las estrategias de desarrollo del país.

La clave para mantener este espacio, según Corrales, es proteger los espacios que están relacionados con disciplinas sobre subsidios, estipulando claramente aquellos que los gobiernos pueden utilizar y aquellos que se consideran violatorios de los acuerdos comerciales. 


\subsubsection{Limitaciones a las importaciones}

A pesar de que son cada vez más limitados los espacios para utilizar el mecanismo de restricciones a las importaciones para proteger algunas actividades económicas nacionales -a través de la aplicación discriminatoria de impuestos y el uso de licencias de importación- existen algunos mecanismos establecidos para el uso de salvaguardias. ${ }^{2}$

El mecanismo de salvaguardias contempla la restricción temporal de las importaciones de un bien, siempre que pueda fundamentarse que la importación de ese bien causa un daño grave a una rama de la producción.

Algunos sectores económicos, como es el caso del sector textil-vestuario nacional de pequeña escala, han sido impactados tras la firma del Cafta, por la entrada al país, sin aranceles, de ropa de segunda mano o con fallas de manufactura. Estas prendas se venden masivamente en el país, lo que ha ocasionado una disminución de la demanda de ropa nacional.

Este es uno de los sectores que, con colaboración del Instituto Nicaragüense de Apoyo a la Pequeña y Mediana Empresa (Inpyme) han sugerido la utilización de una medida de salvaguardia para impedir, al menos temporalmente, la introducción sin aranceles de dichas mercancías.

Se considera apropiado mantener el mecanismo de salvaguardias, e incluso difundirlo más, sobre todo entre aquellos sectores con menor acceso a información, que son por lo general los más vulnerables. Por tanto, esa difusión debe hacerse a través de medios masivos, y no restringirse a la información directa que ofrece el Mific, o a la documentación divulgada en Internet, por ser de limitado acceso.

Dado que las salvaguardias son restricciones temporales, es de suma importancia que a la par se diseñen e implementen medidas que permitan a los sectores protegidos disminuir gradualmente las desventajas productivas con las que se enfrentan, a fin de que, tras el período de la salvaguardia, puedan insertarse a los flujos económicos comerciales.

\subsubsection{Preferencias en las compras públicas}

El mecanismo de compras públicas se ha utilizado de manera diferente en los países desarrollados y en los países en desarrollo. Mientras en los primeros se utiliza para promover el desarrollo tecnológico, así como para introducir estándares ambientales más elevados, en los segundos se dirige más al fomento de las micro, pequeñas y medianas empresas (mipymes) a través de las compras del Estado.

En el Cafta se estableció que ese mecanismo -en el cual los competidores nacionales tienen ventajas sobre los internacionales al ser los segundos excluidos de la competencia por las compras del Estado- tiene para Nicaragua un límite que serán las compras de menos de 58,550 dólares (Delgado-Espinosa, 2008). Esto significa que en la asignación de contrataciones el Estado puede dar un trato preferencial a empresas nacionales, siempre que estas no se excedan del monto antes señalado.

Algunos grupos empresariales, específicamente empresas extranjeras que quedan excluidas de este trato preferencial, se han manifestado a través de las asociaciones que las aglutinan -como es la Asociación Nicaragüense de la Industria Textil y la Confección (Anitec)en contra de la existencia de este mecanismo y del aumento del límite establecido que fue solicitado por el gobierno, alegando falta de transparencia y de competitividad en la asignación de las contrataciones a mipymes.

Sin embargo, sin minimizar la importancia de darle un uso transparente, la existencia de ese mecanismo es crucial para implementar varias iniciativas propuestas en este informe. Por tanto, conviene que los esfuerzos se centren en la definición de los mecanismos de transparencia y de las condicionalidades que se exigirán a las empresas beneficiadas por el mecanismo. Esto con el fin de darle un uso estratégico, que a través de estos 
incentivos condicionados apunte no tanto a la protección permanente de las mipymes -que son a las que mayormente se debería beneficiar con el mecanismo- sino a procurar su competitividad.

Debe por tanto mantenerse el mecanismo de compras públicas, y complementarse con medidas que aseguren la transparencia de su uso, así como su aprovechamiento como incentivo condicionado. De esta manera las mipymes tendrán alicientes adicionales para implementar las estrategias propuestas para éstas: asociatividad, capacitaciones, mejoras tecnológicas, cumplimiento de estándares laborales y ambientales, entre otras; y se disminuirá el riesgo de que el mecanismo promueva el proteccionismo y disminuya la competitividad de las mipymes, u obligue a los consumidores a obtener bienes de mala calidad a mayores precios.

\subsubsection{Protección a la propiedad intelectual}

Tomando en cuenta la necesaria vinculación que existe entre el conocimiento, la tecnología y la competitividad, y las enormes limitaciones que existen en Nicaragua para que muchas empresas, especialmente mipymes, paguen licencias sobre el uso o la adaptación de tecnologías, ocurre que la implementación del Acuerdo sobre Aspectos de Propiedad Intelectual Relacionados con el Comercio (Adpic y Adpic-Plus ${ }^{3}$ ) es uno de los mayores desincentivos para el desarrollo tecnológico de las mipymes. La razón principal es el costo asociado al uso de la tecnología, que para el sector empresarial más vulnerable implica una inversión inalcanzable.

Sin menoscabar la importancia de los derechos de propiedad intelectual para el fomento de la innovación, este acuerdo debe ir ligado a políticas de uso que aseguren que los sectores económicos más vulnerables no quedan excluidos. Es decir, también el conocimiento y la tecnología deben ponerse al servicio del desarrollo sostenible, lo cual implica garantizar el acceso a los medios necesarios para el desarrollo de los actores económicos.
Las restricciones económicas para acceder al conocimiento deben disminuirse.

Por otra parte, debe existir un claro mecanismo de apoyo a las mipymes para que registren sus creaciones y puedan beneficiarse de ellas; pero también para evitar que empresas extranjeras se apropien del conocimiento local. A corto plazo la medida más importante es disminuir los costos por los trámites de registro de marcas y patentes. Igualmente necesario es proveer información sobre la importancia de este registro, y sobre los trámites requeridos para evitar los riesgos que se derivan de no registrar las creaciones nacionales.

\subsubsection{Exportaciones de vestuario}

En términos generales, el nivel de exportaciones de prendas de vestir desde el régimen de zonas francas (RZF), sobre todo al mercado de Estados Unidos, ha sido muy pujante. En los últimos dos años, sin embargo, se observa un decaimiento ocasionado sobre todo por la crisis mundial que ha repercutido en los niveles de demanda de prendas de vestir.

A pesar de las conquistas en materia comercial que se lograron para el sector textil-vestuario de Nicaragua en el marco del Cafta, en la actualidad muchos de los acuerdos han presentado limitaciones que deben analizarse, sobre todo en el momento de negociar nuevos acuerdos comerciales. Los temas más relevantes se presentan a continuación.

\subsubsection{Reglas de origen}

La regla de origen estipulada en el Cafta para el sector "establece que Nicaragua puede exportar a Estados Unidos y disfrutar aranceles preferenciales Cafta, siempre que las prendas de vestir se hayan hecho en el país - o en la región Cafta- de la hilaza en adelante. La materia prima, como el algodón en bruto, lana, fibras e hilo, puede importarse de otros países, sin embargo, todas las etapas del proceso de producción comprendidas del hilado en adelante deben llevarse a cabo en la región" (Portocarrero, A. 2009). 
Considerando que la mayor parte de los tex-tiles que se utilizan en Nicaragua para exportación son de origen asiático, se negoció el uso del mecanismo de los TPL (Trade Preference Level), a través del cual se pueden exportar prendas de vestir con telas que no son de la región Cafta, hasta por una cantidad de metros cuadrados, y continuar disfrutando de los beneficios de los aranceles. Sin embargo, ese mecanismo está condicionado a que se importe una determinada cantidad de tela hecha en Estados Unidos, por la cantidad de TPL utilizada. A este condicionamiento en el uso de los TPL se le llama la "regla uno a uno". ${ }^{4}$

La ventaja en el uso de los TPL es temporal, y sumándole a eso las condicionalidades a las que está sujeto su uso, obliga a plantear el tema como una prioridad en materia de negociaciones comerciales. Tras varios años de la firma del Cafta, la experiencia en Nicaragua es que algunos empresarios no están haciendo uso de los TPL, sino exportando directamente hacia Estados Unidos (sin gozar de los beneficios arancelarios de (afta) prendas de vestir hechas con telas asiáticas.

A juicio de Anitec, la razón de este fenómeno es que las telas que se producen en la región son mucho menos competitivas que las telas asiáticas, y que muchos empresarios obtienen más beneficios al exportar a Estados Unidos con telas asiáticas y pagando aranceles, que exportando con tela regional libre de aranceles y condicionada a la importación de tela de Estados Unidos.

También señalan que la tendencia en la región es a la disminución de la industria textil, y que por tanto en futuras negociaciones comerciales la regla de origen debería modificarse, y estipular que se puedan exportar prendas de vestir de la región Cafta a Estados Unidos, siempre que las mismas se hayan producido en la región, de la tela en adelante, y no de la hilaza en adelante, como está definida hasta ahora.

Este tema es crucial, pues implica alcanzar consensos entre los países de la región en primera instancia, para tener una posición más firme en futuras negociaciones. Dichos consensos son complejos, dado que los intereses de la industria textil de cada uno de los países difieren entre sí.

\subsubsection{Mecanismo de acumulación}

Se considera pertinente continuar impulsando el mecanismo de acumulación ${ }^{5}$ con México y con otros posibles socios. Dicho mecanismo permite ampliar la cadena de suministros del sector, obteniendo insumos de México, así como ampliar el mercado de destino a dicho país.

Sin embargo, conviene hacer una mayor divulgación del mecanismo, de manera que sean visibles las ventajas que ofrece. Ese mecanismo debería dirigirse sobre todo a empresas de menor tamaño, cuyo acceso a este tipo de información es aún escaso.

\subsubsection{Derechos laborales y medio ambiente}

En el Cafta se estableció que la responsabilidad de cumplir los marcos jurídicos nacionales en materia laboral y ambiental recae en el Estado y no en el sector empresarial. Además se decreta que si por falta de monitoreo, de definición de procesos, o de claridad en la información y procedimientos que las empresas deben realizar se afecta negativamente a una empresa extranjera operando en el país, el daño causado podría ser motivo de que esta empresa entable demanda al Estado (Portocarrero, A. 2009).

En el diagnóstico del sector se identifica como un problema significativo en términos ambientales la inefectividad de los mecanismos de monitoreo y evaluación en manos de las instituciones nacionales que rigen los asuntos ambientales, especialmente el Marena. Además, según representantes del sector empresarial, ha habido dificultad y lentitud en la definición de algunos procesos importantes, como lo es el tratamiento de los desechos sólidos.

Según los informantes, una de las razones por las cuales Marena no ha logrado efectuar una eficaz labor de monitoreo y evaluación del cumplimiento de las empresas en materia ambiental es la disminución de los recursos con 
los que dispone. Así, es evidente la necesidad de que, a la par de las negociaciones comerciales en las que Nicaragua se compromete a garantizar el cumplimiento de la legislación nacional en materia ambiental y laboral, se haga también un ajuste en las capacidades de las instituciones que se dedican a esa labor.
Este es uno de los temas cruciales en la Agenda Complementaria del Cafta, y se considera importante mantener e impulsar en futuros espacios de negociación las iniciativas que se plantean en esa Agenda, así como nuevas agendas que surjan de las necesidades del país. 


\section{AYUDA PARA EL COMERCIO}

“... Para que el comercio funcione es esencial que el financiamiento para el comercio esté disponible y accesible". 6

Pascal Lamy, director general de la Organización Mundial del Comercio (OMC), en su discurso del 29 mayo de 2009 en la Reunión Regional de Ayuda para el Comercio en Camboya, reforzó el compromiso que la OMC ha adquirido con los países menos desarrollados, de disminuir los costos que el comercio tiene sobre algunos sectores de sus economías, así como mejorar su inserción en los mercados internacionales mediante apoyos financieros que disminuyan los obstáculos que estos países tienen para el comercio. ${ }^{7}$

Ayuda para el Comercio es un fondo cuyo objetivo es apoyar a los países en desarrollo a mejorar sus capacidades por el lado de la oferta y su infraestructura relacionada con el comercio, para ayudarles a que implementen y se beneficien de los acuerdos de la OMC y, más ampliamente, para expandir su comercio.

El mandato para la creación de ese fondo se estableció en 2005 en la declaración ministerial de la OMC en Hong Kong. Posteriormente se establecieron los mecanismos para su implementación y operativización. Tomando en consideración que los obstáculos para el comercio dependen de la estructura económica de cada país, los criterios para la ejecución del fondo dependen de las demandas de cada uno de los países.

Así pues, cada país debe presentar su estrategia de ayuda para el comercio en función de sus necesidades específicas, mismas que deben estar vinculadas a sus planes de desarrollo y de reducción de la pobreza.

El diagnóstico del sector textil-vestuario nicaragüense muestra los principales obstáculos del país para el comercio, así como las limitaciones existentes para que el comercio sirva a objetivos de desarrollo. A pesar de estar centrado en un sólo sector de la economía, se identifican problemas estructurales con cuya solución podrían beneficiarse distintos sectores económicos.

Muchos de estos obstáculos son coincidentes con las prioridades que se han identificado en las revisiones regionales sobre Ayuda para el Comercio organizadas por la OMC. En la reunión regional de 2007 participaron ministros de finanzas y de comercio, donantes bilaterales, agencias regionales y multilaterales, y representantes del sector privado.

Se presentan a continuación los temas prioritarios de ese foro, mismos que deben incluirse en las estrategias nacionales destinadas a disminuir los obstáculos para el comercio, así como otros temas importantes para el sector textil-vestuario nicaragüense.

\subsection{Infraestructura de Energía y Transporte}

Los temas de energía y transporte (terrestre y marítimo) son de importancia capital para el desarrollo productivo nicaragüense. En ambos temas existen serias deficiencias en el país. ${ }^{8}$ Convendría abordar una estrategia desde una perspectiva regional, que además de mejorar la competitividad de los países centroamericanos, sirva de elemento integrador de la región. Asimismo, en la estrategia de país debe incorporarse el uso de fuentes alternativas de energía, con una visión de sostenibilidad ecológica.

\subsection{Estructura Regulatoria}

Además de la infraestructura física, una estructura regulatoria sólida es esencial para asegurar estabilidad para los inversionistas, así como para garantizar las condiciones para que el comercio sirva a una estrategia de desarrollo sostenible del país.

Son varios los temas que hay que mejorar a este respecto: la definición y agilización de trámites y procedimientos para hacer negocios, específicamente los trámites aduanales 
para la internación y salida de insumos y productos; la obtención de permisos ambientales; la definición de mecanismos para el tratamiento de desechos sólidos y contaminantes; los trámites de legalización de empresas; el registro de marcas; los requisitos de exportación, y otros. ${ }^{9}$

En cuanto a las funciones de monitoreo y evaluación de instituciones tales como Marena y el Mitrab, encargadas del cumplimiento de los marcos jurídicos del país en materia laboral y ambiental, es esencial que se les destinen mayores recursos para fortalecer su quehacer. En el caso de Marena, para ilustrar, se ha registrado en los últimos años una disminución de su presupuesto y un aumento del alcance de sus responsabilidades, al multiplicarse el número de empresas a las cuales debe dar seguimiento la institución.

Por otra parte, hace falta que se creen y se promuevan mecanismos para que el Estado y el sector empresarial puedan compartir información que sea de utilidad para ambos. Por el lado del Estado, es conveniente y saludable que reciba retroalimentación sobre su quehacer, los servicios que puede mejorar y las necesidades del sector empresarial, con el fin de asegurar un mecanismo de mejora constante de sus servicios que propicie mejores condiciones para hacer negocios en el país. Los temas de aduanas, impuestos y permisos son especialmente sensibles para el sector empresarial, por tanto, la retroalimentación que sobre estos temas pueda tener el Estado es un insumo muy útil para mejorar el clima de negocios del país. Para el gobierno, por su parte, recibir información sobre la forma en que el sector afecta positiva y negativamente al país le permite redefinir su política de relaciones con el sector empresarial, así como sus condiciones ante el sector en función de consolidar una estrategia de desarrollo.

Para el sector empresarial es conveniente recibir información clara y accesible sobre la estrategia de desarrollo del país y del sector a fin de contribuir a ello, así como sobre los cambios en la legislación, sobre política macroeconómica y otros temas pertinentes.

\section{3 ¿Formación o Capacitación?}

La formación o capacitación es uno de los escollos para el crecimiento del comercio, según el sector privado. Asimismo, es uno de los principales obstáculos que experimenta Nicaragua para que el comercio genere desarrollo sostenible y equitativo.

La necesidad de transformar la oferta educacional a fin de que responda a los requerimientos del mercado laboral es importante para generar crecimiento, pero no basta para asegurar desarrollo. En este sentido, los cambios en el sistema educativo medio, técnico y superior deben ser un instrumento clave de una política de desarrollo más amplia e integral, de la cual se vea beneficiada la sociedad en su conjunto.

Este es uno de los temas prioritarios y estructurales que deben ser parte de la estrategia del país para disminuir los obstáculos al comercio y el desarrollo. Por tanto, se considera relevante proponer estrategias vinculadas a la formación y capacitación, que puedan financiarse con fondos de Ayuda para el Comercio.

\subsection{Fortalecimiento del Sector Privado}

El sector privado, especialmente aquellas empresas con menor acceso a información, capital y conocimientos, puede ser apoyado por Ayuda para el Comercio en varios aspectos. Además de temas estructurales como la infraestructura física, la capacitación y lo que atañe a logística y regulación, las empresas enfrentan obstáculos a nivel micro que deben tomarse en cuenta.

Se considera estratégico fortalecer la capacitación técnica, a fin de que permita a las mipymes conocer temas relacionados con las exportaciones, como son los estándares de exportación y las reglas de origen que deben cumplirse para hacer uso de los beneficios de los regímenes comerciales. Un gran porcentaje de empresas dedicadas a la confección, especialmente las de menor 
tamaño, requieren de un acceso adecuado a este tipo de información, que les permita adaptar su producción a las exigencias de los mercados internacionales. Esta información debe ir acompañada de apoyos financieros para que las empresas realicen las inversiones necesarias para cumplir con esos estándares.
Dentro del apoyo al sector privado se de-ben incluir estrategias de promoción y diversificación de exportaciones, que permitan que la oferta exportable del país se amplíe: de exportaciones basadas en los recursos disponibles, a exportaciones que además contengan mayor valor agregado y más contenido tecnológico. 


\section{POLÍTICAS ESTRUCTURALES}

Las políticas estructurales abarcan mucho más que lo relacionado exclusivamente con el sector textil y de confecciones. Por tanto, muchas de las recomendaciones del presente informe ya se han incorporado en estrategias diseñadas para otros sectores e incluso en el Plan Nacional de Desarrollo Humano (PNDH). Sin embargo, conviene enfatizarlas porque son esenciales para implementar las estrategias específicas del sector.

A continuación se abordan varios temas: articulación entre el PNDH, la política comercial y la política industrial del país; política de educación; infraestructura; política de atracción de inversiones; participación inclusiva en flujos económicos internacionales; y políticas de financiamiento.

\subsection{Articulación en Política Económica}

Existen importantes diferencias en la estrategia de desarrollo planteada en el PNDH diseñado por el gobierno actual y el Plan Nacional de Desarrollo (PND) del gobierno anterior. La estrategia del PNDH se enfoca mucho menos en la atracción de IED para la exportación -que era parte fundamental de la estrategia del PND - y más en el fortalecimiento industrial que combine una estrategia de sustitución de importaciones con una de apertura comercial.

A pesar de esta importante modificación en el PNDH, no se explicita cómo este cambio afectará la política comercial interna y externa del país, y en específico no se encontraron modificaciones relevantes en la estrategia de negociación del Acuerdo de Asociación de Centroamérica con la Unión Europea en comparación con la negociación del Cafta. Incluso se ha señalado oficialmente que el acuerdo con la UE tiene base Cafta, es decir, contiene una parte de lo ya negociado en el acuerdo de comercio entre Centroamérica y Estados Unidos (Portocarrero, A. 2009).
En lo que atañe específicamente al sector textil-vestuario nacional, a pesar de la importancia que se le ha dado en las negociaciones comerciales, así como en la estrategia de atracción de inversiones, el sector no aparece en la estrategia de desarrollo del país planteada en el PNDH. Esta omisión no sólo crea incertidumbre en los inversionistas, sino también desarticulación en la implementación de la política económica entre las distintas instancias que se relacionan con el sector.

Por la importancia que ha tenido y tiene el sector textil-vestuario en las exportaciones del país y en la generación de empleos, a pesar de la crisis económica mundial, es necesario que se incluya en el PNDH un acápite al respecto, que permita redefinir coherente y articuladamente las estrategias de todas las instituciones relacionadas con el sector.

Sólo en la medida en que exista una postura oficial hacia el sector y que se establezca claramente de qué manera debe insertarse en la estrategia de desarrollo del país será factible diseñar y afinar las políticas, programas y proyectos al respecto.

Especialmente importante es el vínculo entre política comercial y política industrial. Los espacios que el país asegure en el mercado internacional deben estar estrechamente relacionados con la política industrial del país, que es la que determina buena parte de la oferta exportable nacional, de la cual forma parte la industria textil-vestuario. Hasta hace muy poco en Nicaragua estas dos áreas de política estuvieron divorciadas, lo cual se evidencia en la ausencia de una política industrial hasta el año 2008, cuando fue creada. Esta evidente separación debe revertirse, a fin de que la política comercial y la política industrial del país se complementen y se retroalimenten mutuamente. 
Deben establecerse claramente los vínculos entre ambos instrumentos de política, los mecanismos de retroalimentación, los espacios de discusión y los actores que desempeñan el rol de relacionar y asegurar la coherencia de los objetivos de ambos instrumentos. El sector empresarial debe ser uno de estos actores, y deben definirse los espacios para su participación. En esos espacios debe asegurarse también la representatividad de las empresas grandes y de las mipymes.

\subsection{Política de Formación y Desarrollo}

La necesidad de diseñar un régimen de atracción de inversiones que genere empleos masivos está directamente relacionada con los altos índices de desempleo del país y con el bajo promedio de escolaridad de la población. La intensidad de la mano de obra en industrias como la textil-vestuario bajo el modelo que se implementa en Nicaragua suele estar relacionada con bajos niveles tecnológicos y bajos niveles salariales. Es decir, la mano de obra barata reemplaza la inversión de las empresas en tecnología moderna. En contraste, las empresas con mayores niveles tecnológicos emplean mucho menos personal en su proceso productivo. Así pues, una estrategia de desarrollo sostenible debe partir de la preparación de la mano de obra del país, a través de procesos formativos que permitan que ésta se inserte en el mercado laboral con la posibilidad de generar mayor valor agregado, y que, por tanto, las principales motivaciones para invertir en el país no sean la abundancia y la baratura de la mano de obra.

Considerando que los recursos que se invierten en el sector educativo son siempre menores que las necesidades del país en esa materia, es muy importante que la política educativa sea coherente con el PNDH y con sus instrumentos de política. Es decir, los recursos que el Estado invierte en educación deben servir para preparar una oferta de mano de obra que se complemente con la demanda laboral existente, y que además gradualmente vaya atrayendo inversiones de mayor generación de valor agregado.
La poca oferta -y poca demanda- de estudios técnicos muestra la dislocación que existe entre el mercado laboral y el educativo. Acercar ambos mercados no sólo es necesario, sino urgente, dado que el país podrá estar preparado para un modelo de desarrollo sostenible apoyado en el comercio solamente si es capaz de proveer mano de obra capacitada para participar en los eslabones de mayor valor agregado de las cadenas de valor internacionales.

Específicamente para el sector textil-vestuario existe la potencialidad de proveer una oferta laboral con mayor capacitación técnica para suministrar servicios relacionados, como serían la reparación y adaptación de maquinaria industrial y artesanal, estrategias de producción limpia y eco-innovación, estudios de impacto ambiental, servicios logísticos $\mathrm{y}$ otros muchos.

Si bien esta no es una demanda actual de la mayoría de los empresarios, es esencial que exista una estrategia de capacitación dirigida a capacitar la mano de obra en los eslabones de la cadena de producción poco desarrollados en Nicaragua, tales como el diseño y la comercialización, que son a su vez los que mayor valor agregado generan en la cadena.

\subsection{Infraestructura}

Los aspectos de infraestructura que más afectan a la producción nacional en general son de dos tipos: infraestructura física y logística. Cada uno merece especial atención, pues con distintos niveles de esfuerzo podrían ser enormes potenciadores de la producción nacional para consumo interno y de exportación.

\subsubsection{Infraestructura física}

Son tres los temas estratégicos para el sector en términos de infraestructura física: disminución del costo y aseguramiento del abastecimiento de energía; construcción o 
mejora de carreteras para transportar la producción en menos tiempo y a menor costo, y la construcción de un puerto en el Atlántico que permita el transporte de la producción al exterior del país.

Datos de la Comisión Económica para América Latina (Cepal) actualizados a 2003 muestran que la capacidad energética de Nicaragua depende en un $72 \%$ de las importaciones de petróleo, lo que convierte al país en altamente sensible a los precios de éste. A la vez, para el mismo año el país registraba el costo de electricidad más elevado de Centroamérica, con 123 dólares por megavatio/hora (Envío, junio 2005).

Según el analista económico Adolfo Acevedo, una buena parte del problema del elevado costo de la electricidad en Nicaragua tiene que ver con la estructura del mercado, misma que se caracteriza por considerables niveles de concentración monopólica. La volatilidad de los precios del petróleo se ha trasladado al precio de la electricidad nacional, subiendo considerablemente su costo e impactando no sólo al sector textil-vestuario, sino a la economía en general.

La provisión de energía a menores costos, por tanto, es uno de los grandes retos que tiene Nicaragua para lograr una mejor competitividad. Sin embargo, a la par de las estrategias para asegurar el acceso a la energía eléctrica, el país debe invertir en la disminución de su dependencia respecto del petróleo, impulsando proyectos de energía alternativa que, además de reducir los costos de producción de las empresas y de consumo de los habitantes, contribuyan a la preservación del medio ambiente.

En cuanto a infraestructura de transporte, resulta estratégico construir y mejorar carreteras y caminos, además de un puerto en el Atlántico, a fin de disminuir los tiempos de entrega de las exportaciones y utilizar esta ventaja para reorientar la oferta exportable a aquellos bienes y servicios que requieren tiempos de entrega menores. Específicamente para el sector textil-vestuario la disminución en los tiempos de entrega es una ventaja sumamente estratégica, dado que la oferta de textiles y vestuario podría especializarse en prendas cuyo éxito dependa de la rapidez para surtir los pedidos.

Esto se vuelve más importante en tanto un competidor como China entra con fuerza creciente al mercado de Estados Unidos, desplazando la oferta de vestuario tradicionalmente surtida por Centroamérica. En lugar de competir frontalmente con China, cuyos costos de producción son muy inferiores a los de Nicaragua, la estrategia se centraría en el posicionamiento estratégico del país en función de ventajas que no sean las mismas que ofrece China. Una de las ventajas más importantes es la cercanía con Estados Unidos, y por consiguiente, los menores tiempos de entrega.

\subsubsection{Logística}

Muchos de los actores del sector consideran que los temas logísticos son más importantes aún que la infraestructura física. Dentro del aspecto logístico se señala como lo más relevante el elevado costo y tiempo que toman los trámites que deben realizar las empresas para operar en el país y para exportar.

Entre las instituciones cuyos trámites se consideran menos eficientes $-y$ a los que sería prioritario mejorar- se encuentra la Dirección General de Aduanas (DGA), el Marena, y el Mitrab.

Uno de los trámites más importantes para las empresas que están dentro o fuera del RZF son los mecanismos establecidos por el régimen de aduanas para la internación de insumos y salida de productos. Para las empresas que están fuera del RZF, el problema principal son los prolongados tiempos que toma la importación de insumos; en tanto para representantes del sector exportador, el problema se centra en el empeoramiento de los trámites de salida de los contenedores con su producción, dado que cada uno de los contenedores debe pasar inspección, en lugar de hacerse al azar, como ha sido la demanda del sector. 
La agilización de los procesos a cargo de la DGA es importante y estratégica, pues incide directamente en los tiempos de entrega de la mercancía. Es recomendable hacer una revisión exhaustiva de los cambios que se pueden generar en estos procesos, a fin de que se logren los objetivos del sector empresarial y a la vez se garantice la labor de control de la DGA.

En cuanto al Marena, se considera que el trámite para la obtención de permisos medioambientales, los procesos de monitoreo y los mecanismos para el tratamiento de desechos sólidos están poco definidos o son insuficientes. Se considera que no están bien descritos los pasos para obtener los permisos ambientales que la ley exige, y que no se define claramente qué instituciones se encuentran a cargo de qué procesos para otorgar esos permisos. También se señala que es insuficiente la capacidad del Marena para atender las necesidades del sector, así como para realizar debidamente sus funciones de monitoreo y definición de regulaciones.

Para el sector de empresas de menor tamaño, los trámites para legalizar la empresa, el registro de marcas y los requisitos de exportación son los que se consideran más importantes, y en los que se podría trabajar para aportar al desarrollo del sector.

Debe continuarse procurando disminuir el costo y los tiempos para hacer negocios en Nicaragua. Gobiernos anteriores han iniciado proyectos para ese fin, y en general se percibe una mejora en este indicador, sin embargo, la cantidad de trámites que se requieren para abrir una empresa o para exportar siguen siendo muy numerosos, y la información sobre los requisitos suele estar dispersa y confusa. Esto afecta particularmente a las empresas más pequeñas, pues los costos de estos procesos como porcentaje de su estructura de costos son mucho mayores que para las empresas grandes.

Finalmente, además de suministrar información clara y expedita, hay que definir con precisión qué instituciones están a cargo de cada trámite, y cuáles son las funciones y responsabilidades de cada una. Según los informantes, esta falta de claridad en las atribuciones ocasiona grandes pérdidas de tiempo $y$ de dinero.

\subsection{Política de Atracción de Inversiones}

Desde los años 90 la política de atracción de inversiones ha sido muy importante para la política económica del país. La reapertura del RZF y la política de comercio exterior han sido los principales instrumentos de atracción de inversiones impulsados hasta ahora. Son notables los resultados en términos de número de empresas extranjeras instaladas en el país, así como de exportaciones a través del RZF. Sin embargo, conviene hacer algunos cambios en el diseño del RZF a fin de dar un nuevo impulso al sector textil-vestuario, pero sobre todo para asegurar que este importante instrumento responda a una estrategia de desarrollo sostenible para el sector y para el país.

Lo que se propone aquí fundamentalmente es modificar la política de atracción de inversiones, de manera que esté en función de crearencadenamientos y complementariedades en los sectores que promueve, y en función también de atraer inversiones de mayor valor agregado, con más transferencia tecnológica y de conocimientos.

Para ser compatibles con las estrategias propuestas, los incentivos del RZF tendrían que focalizarse en aquellas inversiones que cumplan con los criterios arriba mencionados, es decir, que respondan a objetivos estratégicos del país, de manera que se supere gradualmente el objetivo de atraer inversiones únicamente para generación de empleos.

Para el sector textil-vestuario en concreto, esto implica atraer inversiones que complementen la cadena de producción del sector en aquellas actividades que generan mayor valor agregado, pero que además aseguran mayores derrames de conocimientos y tecnológicos. Este cambio se aplica también a otros sectores de la economía. 
Los incentivos que ofrece el RZF, que son una de las ventajas temporales que Nicaragua ofrece a los inversionistas, deberían condicionarse en función de algunos requisitos que vayan más allá de generar empleos y aumentar las exportaciones. Por ejemplo, los temas ambientales y laborales, no solamente en términos de cumplir las reglamentaciones básicas, sino como estímulos para crear un incentivo adicional en el sector empresarial para invertir en el mejoramiento de su imagen empresarial.

Otro tema que podría promoverse a través de incentivos condicionados es la creación de alianzas entre empresas nacionales y extranjeras, ya sea desde el subcontrato, la complementariedad o el derrame tecnológico. La propuesta no es generar relaciones ficticias, sino utilizar los incentivos de manera que ese relacionamiento sea atractivo para los inversionistas. Las condicionalidades tendrían que diseñarse no sólo para atraer al sector de las grandes empresas internacionales a vincularse con el sector nacional mediano o pequeño, sino también para que el sector nacional se comprometa a cumplir con los criterios de calidad y competitividad requeridos.

El sector empresarial grande tiene un rol importante en la transferencia de conocimientos y tecnología al sector mediano o pequeño. A través de los incentivos que ofrece el RZF podrían diseñarse estrategias que promuevan el apadrinamiento de empresas grandes a empresas pequeñas, en términos de procesos de producción, relaciones de proveeduría, información técnica, diseño, entre otros. En el país ya se han registrado experiencias exitosas de apadrinamiento, como es el caso de la empresa Rocedes hacia la empresa Oscarito's. Esas experiencias podrían propagarse mediante incentivos que ofrezca el RZF a las empresas más grandes, atados a condicionalidades para las empresas más pequeñas.

En ese proyecto de apadrinamiento Rocedes invitó a una parte del personal de Oscarito's para que conocieran y se entrenaran en asuntos de sistemas y líneas de producción, formatos, herramientas, rapidez, calidad, manejo del personal, con el propósito de que Oscarito's adaptara los conocimientos adquiridos a sus necesidades y su tamaño. El resultado, según Scott Vaughn, director de Rocedes, fue muy positivo, y se vio reflejado en un mejor posicionamiento de Oscarito's en el mercado nacional e internacional (Portocarrero, A. 2009).

Otra cosa que hay que modificar en el actual RZF es el mecanismo de intercambios entre las empresas que son parte de ese régimen, dado que presenta limitaciones que disminuyen su nivel de relación. Para efectos de control, las empresas deben registrar como importaciones y exportaciones los bienes y servicios que intercambian entre ellas, lo cual reduce los incentivos para realizar dichos intercambios, y por consiguiente se reduce la generación de complementariedades y encadenamientos a lo interno del RZF.

Considerando que una de las estrategias propuestas es generar mayores sinergias, es importante revisar este mecanismo a fin de que existan mayores estímulos entre empresas del régimen para intercambiar bienes y servicios. Además, con una adecuada política de incentivos las mipymes podrían tomar parte en estos intercambios, y de esta manera insertarse con los menores costos posibles en la cadena de producción del sector.

En términos generales, las empresas adheridas al RZF son de capital extranjero. Esto se explica porque desde un principio el régimen se diseñó para atraer IED. Sin embargo, con el fin de generar mayores relaciones entre las empresas de capital extranjero y las empresas nacionales, y de que los beneficios que el país otorga al sector empresarial sean aprovechados por mayor número de empresas de capital nacional, debe incorporarse al régimen una estrategia de atracción de capital nacional.

Para esto se deben revisar los requisitos vigentes para formar parte del RZF, las barreras de entrada a empresas de capital nacional, los mecanismos de difusión del régimen y de atracción de inversionistas, y modificarlos 
en función del objetivo de incorporar a más inversionistas nacionales en el sector.

Finalmente, es primordial que todas las instituciones encargadas de definir y promover los beneficios que ofrece Nicaragua a la inversión extranjera, entre ellas Pronicaragua, trabajen de manera alineada y definan mecanismos claros de retroalimentación acerca del diseño de una estrategia de atracción de IED acorde con los objetivos del país. Esos mecanismos de retroalimentación deberán generar información de utilidad para el sector empresarial, pero también para el Estado, acerca del desempeño de la inversión instalada en el país.

\subsection{Participación Inclusiva en el Comercio}

Poner el comercio en función del desarrollo pasa por la participación activa de los miembros de la sociedad, tanto de aquellos directamente vinculados con las actividades comerciales como de los que solamente perciben las externalidades positivas y negativas asociadas a éstas. Esta participación solo será efectiva en la medida en que los y las participantes tengan los medios y capacidades necesarias para incidir en las esferas en las que se toman las decisiones que les afectan.

Las propuestas que se mencionan a continuación se enmarcan en la necesidad de crear estas capacidades y de romper con las distintas barreras que existen en Nicaragua para participar en el comercio y en la política económica, y que en específico en el sector textil-vestuario impiden la participación de muchos actores nacionales en los beneficios del comercio internacional.

\subsubsection{Participación en política económica}

Un primer espacio importante es el diseño de la política comercial exterior, y en concreto, las negociaciones de los tratados comerciales internacionales. En el proceso de negociación del Cafta fue evidente que las relaciones de poder a varios niveles determinaron quiénes fueron los ganadores y los perdedores del tratado. "En el Tratado de Libre Comercio entre Estados Unidos, Centroamérica y República Dominicana, el Cafta-DR, el poder se manifestó a tres niveles. El primero, entre país y país, Nicaragua vs. Estados Unidos. El segundo, entre actores como el sector privado y la sociedad civil. Y el tercero, dentro de estos dos grupos” (Carrión, 2009).

La desigualdad de poder entre Estados Unidos y Nicaragua en el marco de las negociaciones es obvia, y no será motivo de análisis en el presente informe. Sin embargo, la desigualdad de poder de negociación entre el sector privado y la sociedad civil, así como a lo interno de ambos grupos es de suma relevancia si se pretende que en dichos acuerdos queden plasmados los intereses de la diversidad de actores sociales, y no sólo de aquellos con capacidad de cabildeo.

Según Carrión, en el acceso a los negociadores del Cafta hubo un desequilibrio entre el sector privado y la sociedad civil. Mientras el sector privado trabajaba hombro a hombro con el equipo negociador -en algunos casos de manera bilateral e informal, asegurando así sus intereses- las propuestas de la sociedad civil fueron canalizadas de manera mucho menos fluida, y la mayoría no fue integrada a la estrategia de negociación nacional.

Estas organizaciones civiles generaron propuestas sobre temas delicados y de gran impacto para el país. La omisión de estas propuestas ocasionó que en muchos de estos temas la posición de Nicaragua quedara en mayor desventaja respecto de Estados Unidos. Es decir, estos espacios fueron cedidos a favor de Estados Unidos a cambio del acceso a mercados de ciertos productos "ganadores" de Cafta. Justamente son los aspectos relacionados con los espacios para política los que formaban parte de las propuestas de la sociedad civil, entre los que se encuentran: subsidios agrícolas, barreras no arancelarias de mipymes, condiciones laborales, estándares ambientales y patentes. 
Además de esta brecha de poder entre el sector privado y las organizaciones de la sociedad civil, en las negociaciones se reflejaron también las brechas entre grandes empresarios y mipymes, así como entre organizaciones de la sociedad civil. El limitado acceso a recursos económicos y a información adecuada son las causas principales de la exigua participación de vastos sectores de la sociedad, entre ellos mipymes, expresa Carrión. Esto generó no sólo sectores ganadores y perdedores, sino a su vez actores ganadores y perdedores a lo interno de los sectores. Los ganadores evidentemente eran aquellos que podían trasladarse a Washington a realizar labores de cabildeo con los negociadores e incluso con congresistas estadounidenses, y que además podían acceder al asesoramiento de expertos en materia comercial. La misma autora lo ilustra de la siguiente manera:

"En cambio, los arroceros y productores de lácteos, en su mayoría pequeños y medianos, no contaban con el mismo nivel de recursos económicos para financiarse los pasajes, viáticos y hoteles para asistir a las rondas que se celebraron en distintas ciudades centroamericanas y en Washington ni para contratar a expertos internacionales. Tampoco tuvieron el amplio acceso a círculos políticos que tuvo la CNPA. Las negociaciones comerciales internacionales eran una novedad para actores como la Cámara Nicaragüense del Sector Lácteo (Canislac). Según Wilmer Fernández, vicepresidente de esta Cámara, el lenguaje técnico de las negociaciones comerciales representó una barrera para la participación de muchos de sus afiliados” (Carrión, 2009).

Por tanto, para corregir esta desigualdad y asegurar la representatividad en las negociaciones comerciales, y en general en la definición de políticas económicas que afectan a extensos grupos de la población, no sólo se deben crear los espacios de diálogo, sino asegurar los recursos y la información para que los grupos en desventaja participen de manera adecuada en dichos espacios.

\subsubsection{Barreras no arancelarias}

Un segundo elemento importante para asegurar la participación extensa de los diversos actores de una sociedad en el comercio es identificar y solucionar las barreras no arancelarias que aún existen, mismas que pueden ser mucho más limitantes que las propias barreras arancelarias.

Como barreras no arancelarias se entienden todos los obstáculos -que no sean arancelarios- que encuentran las empresas para participar en los flujos de comercio internacional. Un ejemplo destacable son las normas de calidad, que de no cumplirse imposibilitan a las empresas la exportación a ciertos destinos.

En el caso del sector textil-vestuario, así como en muchos otros sectores del país, la mayoría de la inversión nacional está conformada por mipymes. Muchas de estas empresas trabajan en la informalidad, no tienen marca registrada, y es muy escaso su acceso a recursos financieros, información e insumos de calidad.

Para disminuir estas barreras el gobierno debe procurar disminuir los costos asociados con la formalización de las empresas, el registro de marca y demás trámites asociados al comercio; diseñar incentivos para que las mipymes estrechen relaciones con empresas nacionales y extranjeras de mayor tamaño; promover la asociación entre las empresas más pequeñas a fin de que tengan un mayor poder de negociación con clientes y proveedores, así como para el acceso a fondos que les permitan alcanzar los niveles de calidad que les darán acceso a los mercados internacionales.

El gobierno podría utilizar mecanismos tales como compras públicas, incentivos bajo el RZF especial para mipymes, programas de formalización, capacitación, asistencia técnica y financiamiento condicionados a la mejora de la calidad y criterios previamente establecidos para el logro de las estrategias del sector. 


\subsubsection{Una agenda para las mujeres}

El proceso de globalización ha cambiado la estructura de los mercados laborales. Las mujeres se han insertado cada vez más al mercado laboral, pero en aquellos eslabones de la cadena de producción de menor valor agregado. Tal es el caso del sector textilvestuario, conformado mayormente por mujeres que realizan básicamente labores de confección.

Las ventajas que esta inclusión ha tenido para las mujeres son importantes pero insuficientes, pues los patrones de desigualdad de género continúan reproduciéndose y visibilizándose en los rangos salariales, en el nivel de complejidad de las actividades que realizan hombres y mujeres, en condiciones laborales inadecuadas en algunas empresas, y en la excesiva carga doméstica que tradicionalmente se asigna a las mujeres y que les significa dobles y triples jornadas laborales.

A este respecto, las estrategias y políticas que se están planteando en el documento de estrategias deben afinarse e implementarse con una visión de género que promueva una participación más justa y equitativa de las mujeres en el mercado laboral.

Hay que seguir abogando por una política nacional de empleos y de salarios que asegure la no discriminación de las mujeres. En el caso del sector textil-vestuario, debe asegurarse la inclusión de las mujeres a mandos medios y directivos, con igualdad salarial, así como una mejora de las condiciones laborales de los mandos menores en las empresas.

Se debe visualizar el aporte de las mujeres a la economía a través del diseño de estadísticas con enfoque de género, donde se pueda apreciar la forma en que contribuyen al PIB del país, no solo desde la formalidad del mercado laboral, sino también por el valor del trabajo doméstico y comunitario que realizan.

En los espacios antes mencionados debe garantizarse la participación de las mujeres que forman parte del sector y de las organizaciones que las representan, tanto para el diseño de políticas económicas que las afectan, como para la disminución de las barreras arancelarias que les limitan su participación en el comercio. Así, en las discusiones de política se deben incluir las agendas de las mujeres, de manera que se aborden los obstáculos que ellas tienen para insertarse de manera más beneficiosa al mercado laboral; y deben diseñarse programas de apoyo dirigidos exclusivamente a mujeres. Existen en Nicaragua evidencias muy positivas de los resultados de programas de crédito dirigidos a mujeres.

Finalmente, la efectividad de estas estrategias depende en gran medida de que se promuevan cambios más profundos y complejos en la sociedad, relacionados con la inequidad de género que atraviesa el espacio público y privado. De no generarse cambios en la distribución del trabajo reproductivo en pro de una mayor equidad entre hombres y mujeres, las mujeres continuarán soportando una carga excesiva, en detrimento de su salud física, mental y emocional.

Evidentemente estos cambios no se generan de la noche a la mañana, pero con una estrategia seria, explícita y comprometida se podría mejorar considerablemente la situación de muchas mujeres nicaragüenses.

\subsection{Políticas de financiamiento}

El acceso a recursos financieros adecuados es uno de los problemas que más afectan al sector privado nacional de todos los tamaños, y constituye una de las principales barreras no arancelarias de las mipymes. No es un problema exclusivo del sector textilvestuario, y por tanto se aborda como una política estructural.

El Banco de Fomento Estatal (Produzcamos) cuyo objetivo principal es flexibilizar el crédito directo a los sectores productivos, supuestamente iniciará operaciones en dici-embre de 2009. A pesar de ser ésta una de las principales necesidades del país, ampliamente reconocida por diversos sectores, el manejo político que se está realizando acerca de ese banco ha generado dudas sobre su función y su futuro manejo. 
Sin embargo, tras casi dos décadas de que se privatizó la banca en Nicaragua el hecho de que exista una entidad bancaria estatal implica un cambio importante en la visión y ejecución de la política económica en el país, en donde el Estado tiene un rol más activo en el fomento de la producción nacional.
Aunado a Produzcamos, se debe promover en la banca privada el diseño de instrumentos dirigidos al sector industrial, que se adecúen a las necesidades de financiamiento de empresas de distintos tamaños. Hay que tomar en cuenta que los tiempos de retorno de muchas actividades industriales son más prolongados que los de muchas actividades comerciales. 


\section{CONCLUSIONES GENERALES}

Se observa una importante correspondencia entre las necesidades estructurales del país -en términos de mejora de la oferta- y las prioridades del mecanismo de Ayuda para el Comercio ofrecido por la OMC.

Esta coincidencia se debe capitalizar de modo tal que se asegure el aprovechamiento de ese mecanismo, y se enrumbe el país hacia una estrategia de desarrollo apoyada en el comercio, como proponen las Edsac.

Son cruciales los diagnósticos sectoriales co-mo el efectuado para el sector textilvestuario, que permiten identificar los elementos claves que hay que considerar en las estrategias de desarrollo nacional y sectorial.

Además, esos diagnósticos permiten identificar los espacios para política que deben

garantizarse para poder implementar las estrategias de desarrollo sectoriales y del país. Considerando que muchos de estos espacios se definen a través de negociaciones comerciales, es necesario que para dichas negociaciones se realicen ejercicios que permitan construir participativamente las agendas de negociación de cada uno de los sectores.

Finalmente, con los fondos de Ayuda para el Comercio la región centroamericana tiene la gran oportunidad de presentar estrategias sectoriales regionales que permitan utilizar con mayor eficiencia los fondos disponibles, y además impulsar la integración de la región, a partir del reconocimiento de sus debilidades y potencialidades. 


\section{NOTAS}

Otexa: http://otexa.ita.doc.gov/.

2 Según la OMC, una salvaguardia es la restricción temporal de las importaciones de un producto.

3 Adpic y Adpic-Plus son acuerdos más restrictivos que se han negociado en acuerdos bilaterales.

4 En la "regla uno a uno" se estipula que en el primer año, por el uso de 100 millones de metros cuadrados equivalentes (SME) de tela de terceros países en la producción de pantalones se deben importar 20 millones de SME de tela originaria de Estados Unidos, de las categorías 347 y 348 -pantalones de vestir largos y cortos para hombres, niños, niñas y mujeres-, aumentando la cantidad en 10 millones de SME anuales hasta alcanzar en el cuarto año los 50 millones de SME. La regla se mantiene en 50 millones de SME hasta el año décimo, en que se termina la concesión de los TPL (Carrión 2009, citando a Traversari).

5 El mecanismo de acumulación con México consiste en que Centroamérica y República Dominicana pueden cortar y confeccionar prendas de tejido plano con materia prima de México y exportar al mercado de los Estados Unidos libre de arancel, por un volumen no mayor a los 100 millones de SME durante el primer año; a la vez, se podrán cortar y confeccionar prendas con materia prima de Estados Unidos y exportar a México libre de arancel, por un volumen máximo de 70 millones de SME durante el primer año. Del segundo año en adelante, el límite se puede aumentar hasta alcanzar un total de 200 millones de SME anuales (Directorio Textil Exportador CNZF, 2008, citado en Portocarrero, A. 2009).

6 Pascal Lamy, director general de la OMC, 29 de mayo 2009 (en traducción de la autora).

7 Párrafo 57 de la declaración ministerial de la OMC del 18 de diciembre de 2005 en Hong Kong.

8 Véase la sección 3 del capítulo IV del presente informe.

9 Véase el acápite 3.2 del capítulo IV del presente informe. 


\section{BIBLIOGRAFÍA CONSULTADA}

Acevedo, A. (2005). “Crisis energética: Aquí está el detalle”. Revista Envío 279. Managua. Nicaragua.

Alfaro, A. \& Ortiz, F. (2007). La industria textil vestuario y sus implicaciones socio-ambientales en Nicaragua. Centro Humboldt. Managua, Nicaragua.

Alonso, E. \& Flores, G. \& Solórzano, O. \& Rosses, M. (2001). Bases para la política industrial de Nicaragua. United Nations Industrial Development Organization - Cámara de Industria de Nicaragua. Managua, Nicaragua.

Blandón, P. (2007). Tomo II Plan de Acción: Política de Industrialización de Nicaragua. Ministerio de Fomento Industria y Comercio. Managua. Nicaragua.

Burga, E. (2009). "Estrategias para la reconversión de la industria textil y confecciones en Guatemala y Nicaragua", International Center for Trade and Sustainable Development, Competitiveness and Development Programme, Issue Paper No. 8, Geneva.

Carrión, G. (2009) “Trade, Regionalism and the Politics of Policy Making in Nicaragua”, Markets, Business and Regulation, Programme Paper Number 5, UN Research Institute for Social Development, Geneva, Switzerland.

Comisión Nacional de Zona Franca (2008). Directorio Industrial Nicaragua 2008. Managua. Nicaragua.

Corrales, W. (2007). Discusión conceptual sobre las estrategias de desarrollo sostenible apoyadas en el comercio. Comisión Económica para América Latina. Santiago de Chile. Chile.

Delgado, A. \& Espinosa, I. "Tratado de Libre Comercio entre Centroamérica, Estados Unidos y República Dominicana. DR-Cafta” (versión amigable). Fundación Internacional para el Desafío Económico Global. Managua, Nicaragua.

Gobierno de Nicaragua (1991). “Normas Jurídicas - de Nicaragua: Zonas Francas Industriales de Exportación” (1991). La Gaceta 221. Managua, Nicaragua.

Gobierno de Nicaragua (2008). "Plan Nacional de Desarrollo Humano 2008-2012: Borrador 0". Managua. Nicaragua.

Mific (2007). La Política Comercial Externa de Nicaragua 1990-2007. Ministerio de Fomento Industria y Comercio. Managua. Nicaragua.

Portocarrero, A. (2009). "Sector textil y confección y desarrollo sostenible en Nicaragua". International Center for Trade and Sustainable Development. Issue Paper No. 7, Competitiveness and Development Programme, Geneva, Switzerland.

Solá, R. (2008). Estructura económica de Nicaragua y su contexto centroamericano y mundial. Hispamer-Universidad Centroamericana. Managua. Nicaragua.

World Trade Organization, International Development Bank (2007). Mobilizing Aid for Trade: Focus Latin America and the Caribbean. Report and Recommendations.

World Trade Organization, Organization for Economic Cooperation and Development (2007). Aid for Trade at a Glance 2007: First Global Review. 


\section{www.ictsd.org}

Otras publicaciones de ICTSD Programa sobre competitividad y desarrollo sostenible:

- Hoja de ruta para el sector textil y confección y el desarrollo sostenible en Guatemala. Documento de Trabajo No.1 por Pedro Prado et al, 2009.

- Estrategias para la reconversión de la industria textil y confecciones en Guatemala y Nicaragua. Documento de Fondo No.5 por Eduardo Burga Bartra, 2009.

- Looking for a meaningful Duty Free Quota Free Market Access Initiative in the Doha Development Agenda. Issue Paper No.4 by David Laborde, 2008.

- Impact of Origin Rules for Textiles and Clothing on Developing Countries. Issue Paper No.3 by Munir Ahmad, 2007.

- Special and Differential Treatment for Small and Vulnerable Countries Based on the Situational Approach. Issue Paper No. 2 by Werner Corrales-Leal, Felipe Baritto, and Sarah A. Mohan, 2007.

- Basic Concepts and Proposals on the use of Policy Spaces in Trade-supported Strategies for Sustainable Development. Issue Paper No. 1 by Werner Corrales-Leal, 2007.

\section{Sobre ICTSD}

Fundado en 1996, el Centro Internacional para el Comercio y el Desarrollo Sostenible (ICTSD, por sus siglas en inglés) es una organización no gubernamental sin fines de lucro basada en Ginebra. Por medio de la provisión de información, creación de una red de contactos, promoción de diálogo, investigación y construcción de capacidades, el Centro persigue dos objetivos principales. Por un lado, empoderar a las partes involucradas en la política comercial y por otro, influir en el sistema multilateral de comercio a fin de promover el desarrollo sostenible. 